\title{
Efecto de la administración subcrónica de glucosamina oral en la regulación del peso corporal, glucemia y dislipidemias provocada por una dieta hipercalórica en rata Wistar
}

\author{
Effect of subchronic oral administration of \\ glucosamine in the regulation of body \\ weight, glycemia and dyslipidemia \\ induced hypercholesterolemic
}

Wistar rat

Cornelio BARRIENTOS ALVARADO'

Jorge SÁNCHEZ VÁZQUEZ²

María Atanasia Silvia CÁRDENAS OSCOY²

Osvaldo GARRIDO ACOSTA ${ }^{1}$

Liliana ANGUIANO ROBLEDO'

\section{RE S U M E N}

\section{Objetivo}

Este estudio evaluó el efecto de la glucosamina oral en el sobrepeso y dislipidemia provocada por una dieta hipercalórica en ratas.

\section{Métodos}

En 4 grupos de ratas Wistar: alimentados con dieta comercial para roedores y agua de beber sin grupo de control y con glucosamina (500 mg/kg-1 por día) grupo glucosamina y con dieta hipercalórica enriquecida al $24 \%$ (g/g) compuesta por manteca de cerdo y agua de beber sin grupo hipercalórico y con glucosamina grupo hipercalórico + grupo glucosamina, durante 22 semanas, se evaluaron el peso corporal, grasa abdominal, niveles de glucemia, triglicéridos, colesterol total y lipoproteínas de alta densidad en suero.

1 Instituto Politécnico Nacional, Escuela Superior de Medicina, Departamento de Fisiología. Plan de San Luis y Díaz Mirón, s/n., Col. Casco de Santo Tomas, Delegación Miguel Hidalgo, 11340, México, DF, México. Correspondencia/Correspondence to: C BARRIENTOS ALVARADO. E-mail: <cornelio_barrientos@yahoo.com.mx>.

2 Escuela Superior de Enfermería y Obstétrica, Departamento de Posgrado. Ciudad de México, DF, México. 


\section{Resultados}

Se observó un aumento del peso corporal y glucemia en suero con dislipidemias en el grupo con dieta hipercalórica grupo hipercalórico versus grupo de controle $(p<0.001)$; al administrarse glucosamina para esta misma dieta grupo hipercalórico + grupo glucosamina se minimizaron los efectos presentados, disminuyendo la cantidad de grasa abdominal y los niveles del perfil lípido en suero $(p>0.05)$ y regulándose el peso corporal, las lipoproteínas de alta densidad y la glucemia basal $(p<0.05)$.

\section{Conclusion}

La glucosamina reguló el peso corporal y la glucemia en sangre y minimizó las dislipidemias provocadas por la dieta hipercalórica, favoreciendo el aumento de colesterol lipoproteínas de alta densidad en las ratas. No afectó el peso corporal y el metabolismo lipídico cuando se administró con dieta comercial.

Palabras ilave: Glucemia. Glucosamina. HDL-colesterol. Peso corporal.

\section{A B S T R A C T}

\section{Objective}

This study evaluated the effect of oral glucosamine on overweight and dyslipidemia caused by a high-fat diet in rats.

\section{Methods}

Four groups of Wistar rats: fed with commercial rodent food and drinking water without (control group) and with glucosamine $(500 \mathrm{mg} \mathrm{kg-1}$ per day) and a high-fat diet enriched with $24 \%$ (g/g) butter pork and drinking water without and with glucosamine, for 22 weeks; the body weight, abdominal fat, blood glucose, triglycerides, total cholesterol, and high density lipoprotein in serum were evaluated.

\section{Results}

Body weight gain, increased blood glucose levels and dyslipidemia were observed in the high-fat diet group versus the control group $(p<0.001)$. When glucosamine was administered the same diet the effects were minimized, with a decrease in the amount of abdominal-fat and lipid profile levels in serum ( $p>0.05)$, regulated body weight, and high density lipoprotein and glycaemia $(p<0.05)$. The glucosamine did not affect body weight and lipid metabolism in rats when administered with a normal diet.

\section{Conclusions}

Glucosamine regulated the body weight blood glucose and dyslipidemia caused by a high-fat diet, favoring increased high density lipoprotein cholesterol in rats. It did not affect body weight and lipid metabolism when administered with commercial food.

Indexing terms: Blood glucose. Glucosamine. Cholesterol, HDL. Body weight.

\section{INTRODUCCIÓ N}

Durante la alimentación, la capacidad de almacenar energía en forma de grasa o Tejido Adiposo (TA) se utiliza para cuando la ingesta de alimentos es escasa. El TA constituye uno de los órganos clave en la homeostasis energética, el cual regula sus propias características funcionales y morfológicas de acuerdo con las condiciones fisiológicas o patológicas predominantes, incluida la homeostasis asociada al sobrepeso y la obesidad'. Como consecuencia adversa, la capacidad de almacenar grasa tiene con frecuencia un valor negativo, debido al consumo excesivo por la dieta ${ }^{2}$, con secuelas metabólicas y otros estados hiperlipidémicos ${ }^{3}$, entre los cuales se detectan una concentración elevada de Triglicéridos (TG ${ }^{4}$, alteraciones del ciclo metabólico de los Ácidos Grasos Libres (AGL), colesterol total corporal y Lipoproteínas de Baja Densidad (LDL, Low Density Lipoprotein) y de Muy Baja Densidad (VLDL, Very Low Density Lipoprotein) en sangre.

Las alteraciones en el metabolismo de las lipoproteínas (dislipidemias) se describen como factor de riesgo de enfermedades del síndrome 
metabólico ${ }^{5-8}$; una de las más frecuentes es la hipoalfalipoproteinemia, la cual se distingue por la reducción del Colesterol de Alta Densidad (HDL, High Density Lipoprotein) y la transformación de las lipoproteínas LDL en su fenotipo denso, con poco colesterol9,10.

La prevalencia de estas enfermedades metabólicas en humanos hace urgente la necesidad de estudios para el entendimiento de sus causas y tratamientos, mecanismos de progresión, métodos de diagnóstico e intervenciones terapéuticas sobre estas patologías, enfocados inicialmente en modelos animales viables que simulan los aspectos primordiales y brindan el conocimiento de nuevas sustancias para su tratamiento ${ }^{11,12}$, modelos útiles en la comprensión y evaluación de los mecanismos implicados en el curso temporal de las patologías, ya que presentan también un desarrollo de sobrepeso y obesidad, hiperglicemias con resistencia a la insulina y dislipidemias con hipertrigliceridemia, hipercolesterolemia y reducción de la relación HDL/LDL, lo que ha conllevado nuevos estudios del impacto de la dieta en el desarrollo de complicaciones, como el síndrome metabólico ${ }^{1,13}$.

Estudios con roedores con dietas ricas en carbohidratos, grasas o combinaciones que imitan los hábitos nutricionales de diferentes poblaciones humanas, diferentes combinaciones y cantidades de carbohidratos (entre $10 \%$ y $30 \%$ ) y grasas (entre $20 \%$ y $40 \%$ ) tuvieron como resultado un incremento del peso corporal, un aumento de la grasa abdominal, hipertrigliceridemia, hiperglucemia, hiperleptinemia, resistencia a la insulina y un incremento de los ácidos grasos libres circulantes ${ }^{11,13,14}$, concluyendo que las dietas altas en carbohidratos y lípidos generan en los roedores la mayoría de las complicaciones presentes en el síndrome metabólico humano.

Estos modelos son probablemente la mejor manera de estudiar la fisiopatología y las complicaciones ejercidas por una dieta hipercalórica así como la alternativas de tratamientos con sustancias, demostrando efectos favorables en la disminución de peso, en el tejido adiposo y el control de las dislipidemias, teniendo como finalidad equilibrar la concentración del LDL, reducir la de triglicéridos y aumentar la del HDL, con inhibidores de la síntesis hepática del colesterol, como las estatinas o con fibratos, para reducir la síntesis de las lipoproteínas VLDL a través del el hígado, e incrementar la de las $\mathrm{HDL}^{15,16}$.

Los estudios sugieren que, al reducir la concentración de colesterol LDL incluso por debajo de los parámetros recomendados, disminuyen los eventos como síndrome metabólico, siendo los fibratos en especial los que modifican las LDL al patrón menos denso (con más colesterol) y elevan la concentración de colesterol HDL, tres efectos necesarios para disminuir las dislipidemias ${ }^{16}$.

Recientemente se ha confirmado que la administración aguda en modelos animales de otras sustancias naturales como derivados de la glucosamina, usada en el tratamiento de la osteoartritis como suplemento alimenticio ${ }^{17}$, reportan efectos en la pérdida de peso y eficacia en la aterosclerosis en animales de laboratorio ${ }^{18,19}$. Si bien se conoce que la osteoartritis o enfermedad articular degenerativa es uno de los padecimientos más importantes de este siglo, con un número cada vez mayor de de personas que la presenta ya que, debido al sobrepeso u obesidad, las personas aceleran el desgaste natural de la articulación, se pone en cuestión si la administración crónica de la glucosamina para el tratamiento de la osteoartritis resulta adyacente al tratamiento de la regulación del peso corporal y por ende de las dislipidemias, reportándose que propician beneficios limitados según el tipo de dieta involucrada con su administración y efecto en el sobrepeso ${ }^{20,21}$.

Sin establecerse hasta el momento un estudio que evalúe la eficacia de la glucosamina en los efectos contra el sobrepeso corporal y las dislipidemias producidas por una dieta hipercalórica, como las consumidas frecuentemente en países industrializados y en personas con osteoartritis, la finalidad de este estudio fue evaluar la eficacia de la glucosamina oral en la regulación del peso 
corporal y las dislipidemias en ratas a través de una dieta hipercalórica.

\section{MÉ T O D O S}

Treinta y dos ratas macho cepa Wistar (200 \pm 30 g), divididas en 4 grupos de 8 animales cada uno y alojadas en jaulas metálicas de $20 \mathrm{x}$ $30 \times 18 \mathrm{~cm}$, se colocaron individualmente en condiciones de laboratorio, con comida y agua ad libitum. Las jaulas se instalaron en bastidores, en modo tal de aislar el contacto auditivo y olfativo, de regular la temperatura $\left(22^{\circ} \mathrm{C} \pm 2^{\circ} \mathrm{C}\right)$ y de exponer los animales a 12 horas de ciclos de luz-oscuridad (07a.m. - 07:00p.m.). Después de 2 semanas de adaptación estos recibieron alimento y agua ad libitum durante 22 semanas de acuerdo al siguiente diseño experimental: Grupo Control (GC): se alimentó con una dieta comercial (Rodent Laboratoty Chow 5001. PMI, Richmond, IN) contenido de $4.5 \%$ de grasa, $23 \%$ de proteína y $46.5 \%$ de carbohidratos, derivando un contenido energético de $3.27 \mathrm{Cal} / \mathrm{g}$, y agua pura para beber. Grupo Hipercalórico $(\mathrm{GH})$ : se alimentó con una dieta comercial enriquecida con grasa saturada: contenido de $23.6 \%$ de grasa de manteca de cerdo, $18.4 \%$ de proteína y $37.2 \%$ de carbohidratos, derivando un contenido energético de $4.41 \mathrm{Cal} / \mathrm{g}$. (48,1\% de la energía obtenida de las grasas) ${ }^{22}$, y agua pura para beber. Grupo Glucosamina (GGlcN): se alimentó con una dieta comercial al igual que el grupo control y glucosamina disuelta en el agua de beber (D+Glucosamine hydrochoride, Sigma-Aldrich St. Louis, Missouri) a una dosis de $500 \mathrm{mg} / \mathrm{kg}^{-1}$ al día, una dosis mayor no tóxica al equivalente para el tratamiento de humanos con osteoartritis 1500 mg por día ${ }^{23}$. La variación de las dosis fue de 75 mg a 200 mg por día a causa del aumento de peso de los animales, sin variar el consumo de agua. Grupo Hipercalórico+Glucosamina $(\mathrm{GH}+\mathrm{Gl} \mathrm{CN})$ : alimentado con una dieta comercial enriquecida con grasa saturada igual al $\mathrm{GH}$ y glucosamina disuelta en el agua de beber como el GGIcN.
Los procedimientos experimentales siguieron las recomendaciones del Consejo Mexicano para el Cuidado Animal (NOM-062ZOO-1999) y fue aprobado por el Comité de Ética y Bioseguridad del Instituto Politécnico Nacional de la Escuela Nacional de Ciencias Biológicas.

\section{Mediciones realizadas}

Ingesta de alimento y peso corporal: medidos diariamente y dos veces por semana respectivamente, utilizando una balanza analítica.

Glucemia basal (Glu): se midió semanalmente, después de un ayuno de 12 horas, a través el método del glucómetro OPTIUM XCEED (Optium MediSense, Abbot Lab, USA) de una gota de sangre obtenida de la cola de la rata.

Niveles basales Triglicéridos (TG), Ácidos Grasos Libres (AGL), Colesterol Total (Col T), y Lipoproteinas (Colesterol) de Alta Densidad (HDL): se midieron en suero obtenido de muestras de sangre colectadas y centrifugadas a $5000 \mathrm{rpm}$ por 15 minutos a $4^{\circ} \mathrm{C}$, en las semanas 1,10 y 20 del tratamiento. Por el método enzimáticocolorimétrico con kit comercial: TG (Randox Laboratories, Crumlin, United Kingdom), AGL (como Non Esterified Falty Acids [NEFA]: Wako Chemicals, Neuss, Germany), Col T y HDL, (Spinreact, Espanã).

Cuantificación de la grasa abdominal (Gra-Abd): al final del tratamiento los animales se sobreanestesiaron con pentobarbital sódico $120 \mathrm{mg} / \mathrm{kg}$ de peso (Abbott Lab, México SA de $C V$, México) vía intraperitoneal y se sumó la grasa intraperitoneal, visceral y epididimal con la ayuda de una balanza semianalítica ${ }^{24}$.

Se calcularon los contenidos de lipoproteínas (colesterol) baja densidad y muy baja densidad, con la fórmula de Friedwald ${ }^{25}$.

Análisis estadístico: se usó el Análisis de Varianza (Anova) de dos vías para las variables TG, AGL, Col T, LDH, VLDL, HDL, Glu y Gra-Abd y el análisis Anova de medidas repetidas para las variables. Ingesta de alimento y peso corporal, 
utilizando la prueba de Tukey para comparar las medias de cada grupo. Asimismo, se empleó la prueba de Pearson para evaluar la correlación de grasa abdominal y las variables de perfil lipídico de los diferentes grupos de estudio. Los factores evaluados fueron la dieta y glucosamina, considerándose una $p<0.05$ como estadísticamente significativa con el programa Statistical Package for the Social Sciences (SPSS) versión 20.

\section{RESULTADOS}

\section{Efectos en la ingesta de alimento y peso corporal}

Después de 20 semanas de tratamiento, el consumo energético no mostró diferencia a lo largo del este para ningún grupo de estudio. El peso corporal fue significativamente mayor en el grupo con dieta hipercalórica $(\mathrm{GH})$ hasta la semana $15(p<0.02)$ y $20(p<0.04)$ con respecto al resto de los grupos (Figura 1A), así como para el grupo con dieta hipercalórica más glucosamina $(\mathrm{GH}+\mathrm{GlcN})$ en la semana $20(p<0.03)$ con respecto al GC y el GGlcN. Sin embargo, el peso corporal verificado entre los grupos con dieta hipercalórica ( $\mathrm{GH}$ versus $\mathrm{GH}+\mathrm{GlcN}$ ) mostró una diferencia significativa entre ellos a partir de la semana $15 \mathrm{y}$ se mantuvo hasta la semana $20(p<0.04)$ (Figura $1 \mathrm{~A})$. Es observable que la glucosamina minimizó el efecto de la dieta hipercalórica en el aumento de peso corporal a medida que se administraba el tratamiento. Con respecto a los grupos con dieta normal (GC y GGlcN), se observó que la administración de glucosamina no afecta el peso corporal ( $p>0.05$ ); por lo tanto, la ganancia de peso fue mayor para el grupo con dieta hipercalórica $(\mathrm{GH}$ y $\mathrm{GH}+\mathrm{GlcN})$. Se observó asimismo una tendencia menor en el grupo que recibió glucosamina durante el tratamiento; a pesar de que la diferencia entre ellos no fue significativa, esta fue importante con respecto a los grupos tratados con dieta normal (GC y GGlcN), especialmente si recibieron glucosamina $(p<0.001)$, destacándose que la glucosamina disminuye la ganancia de peso corporal sin importar la dieta administrada (Figura 1B).

\section{Efectos en el contenido de grasa abdominal, NEFA y glucemia en suero}

Al final del tratamiento - semana 22 -, los porcentajes de depósitos de grasa abdominal en relación al peso corporal fueron mayores significativamente en los grupos con consumo de
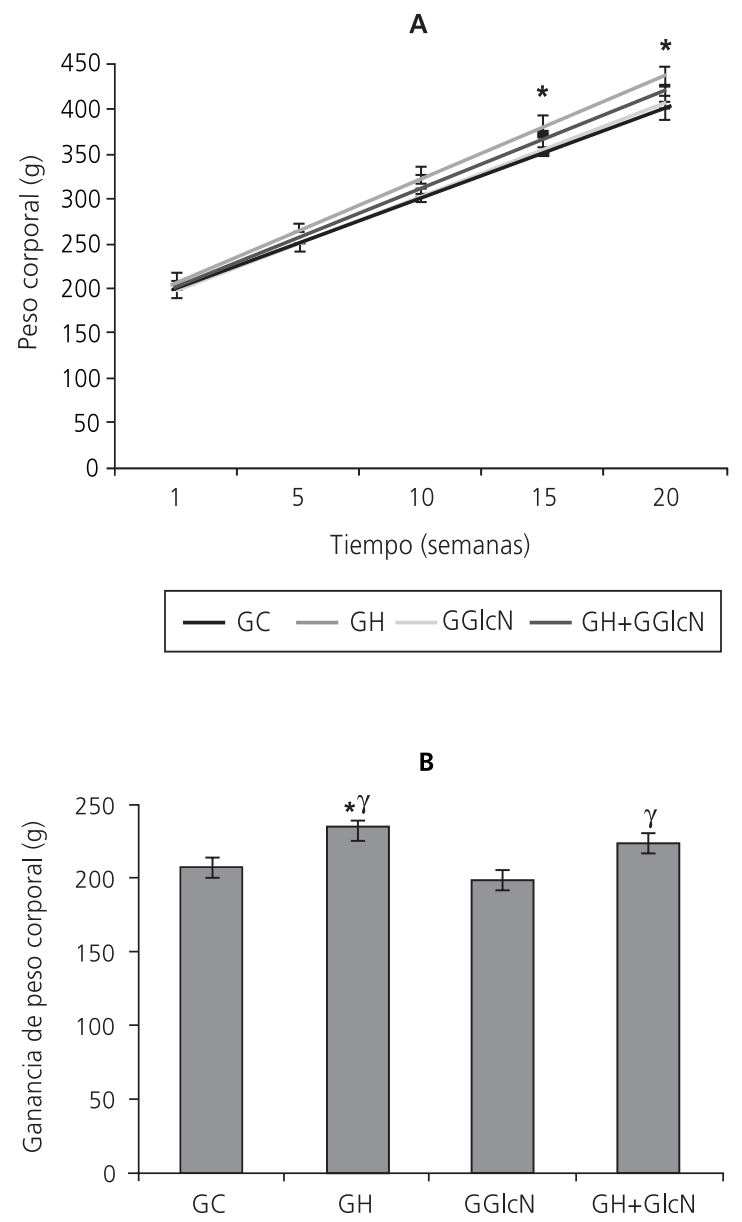

Figura 1. (A) Peso corporal y (B) Ganancia de peso de ratas machos sometidas a una dieta comercial (Grupo de Controle [GC]) e hipercalórica (Grupo Hipercalórico [GH]); con administración de 500 mg/kg de glucosamina respectivamente (Grupo Glucosamina [GGlcN] y GH+GlcN) en el agua de beber, durante 20 semanas.

Nota: Valores se expresan como la media \pm el error estándar $(n=8)$. ${ }^{*} p<0.05$ versus grupo control; ${ }^{\gamma} p<0.05$ versus grupo glucosamina. En cada tiempo de acuerdo a la prueba análisis de varianza para medidas repetidas. 
dieta hipercalórica con y sin glucosamina (GH y $\mathrm{GH}+\mathrm{GlcN}$ ) con respecto al grupo control (GC) $(p<0.03)$, observándose que el grupo que recibió glucosamina minimizó el contenido de grasa abdominal para la dieta hipercalórica y hubo una diferencia importante con respecto al grupo con dieta hipercalórica ( $\mathrm{GH}$ vs GH+GGIcN). Esta diferencia fue más grande con respecto al grupo con administración glucosamina con una dieta normal (GH vs GGlcN) $(p<0.02)$ (Figura $2 A)$. En relación al efecto de la glucosamina (Figura $2 \mathrm{~A}$ ) en dietas normales, esta no alteró, ni tiene efecto sobre el contenido de grasa abdominal, así como tampoco mostró diferencias con respecto al grupo control (GGlcN vs GC) ( $p>0.05$ ).

Los niveles de ácidos grasos cuantificados como NEFA fueron mayores en los grupos con dieta hipercalórica con y sin glucosamina (GH y $\mathrm{GH}+\mathrm{GlcN}$ ) con respecto a los grupos con dietas normales: grupo control $(\mathrm{GC})(p<0.001)$ y grupo con glucosamina (GGlcN) $(p<0.01)$ (Figura 2-B). La glucosamina no altera los niveles de ácidos grasos libres cuando se administra con dietas normales.

Los niveles de glucemia basal fueron mayores significativamente entre el grupo con dieta hipercalórica $(\mathrm{GH})$ con respecto al resto de los grupos estudiados $(p<0.007)$ (Figura $2 C)$. Se observa que la glucosamina regula la glucemia basal sin importar la dieta administrada.

Al final del tratamiento fue demostrado que la glucosamina en el agua de beber minimizó los efectos de la dieta hipercalórica, disminuyendo el contenido de grasa corporal, los ácidos grasos libres y la glucemia en suero.

\section{Efectos en el perfil lipídico por administración de dieta hipercalórica y/o glucosamina}

Como se esperaba, los efectos adversos de la dieta hipercalórica fueron muy característicos en el grupo con este tipo de dieta $(\mathrm{GH})$ sobre las dislipidemias. En general, se verificaron efectos menos adversos en el grupo al que se le brindó
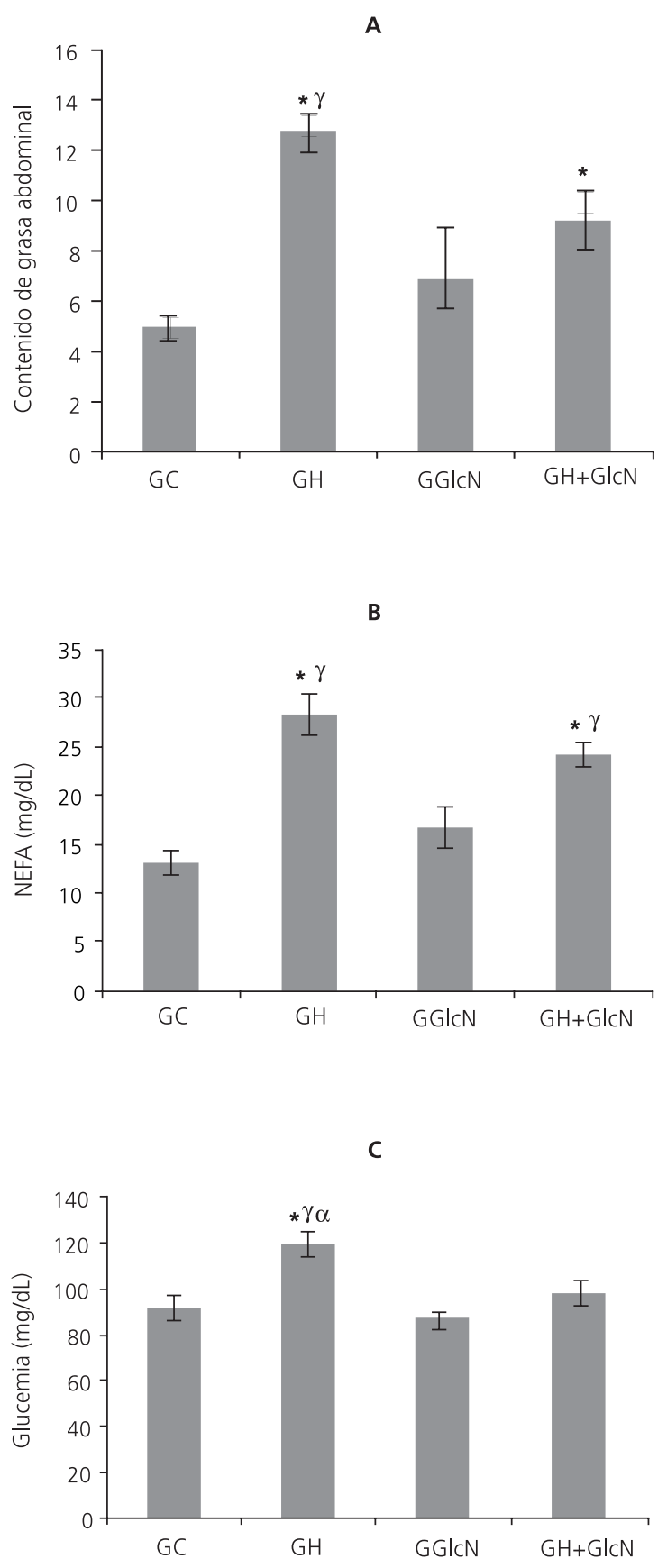

Figura 2. (A) Grasa abdominal ( $\mathrm{g} / 100 \mathrm{~g}$ de peso corporal), (B) Non Esterified Fatty Acidis (NEFA) en suero y (C) glucemia en sangre de ratas machos sometidas a una dieta comercial (Grupo de Controle [GC]) e hipercalórica (Grupo Hipercalórico [GH]); con administración de 500 $\mathrm{mg} / \mathrm{kg}$ de glucosamina en el agua de beber respectivamente (Grupo Glucosamina [GGlcN] y GH+GlcN), al final del tratamiento de 20 semanas.

Nota: Valores expresados como la media \pm el error estándar $(n=8)$. ${ }^{*} p<0.05$ versus grupo control; ${ }^{\gamma} p<0.05$ versus grupo glucosamina;

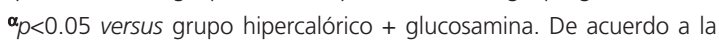
prueba Analisis de Varianza (Anova) de dos vías. 
glucosamina junto a esta dieta hipercalórica $(\mathrm{GH}+\mathrm{GlcN})$, existiendo una diferencia significativa al minimizarlo solo en la variable del índice VDL HDL. Los niveles de triglicéridos, colesterol Col T y lipoproteínas LDL, VLDL en suero fueron mayores para los grupos con dieta hipercalórica ( $\mathrm{GH}$ y $\mathrm{GH}+\mathrm{GlcN})(p<0.001)$ con respecto a los $\mathrm{GC}$ y el grupo con glucosamina (GGlcN) (Tabla 1).

Con respecto a las lipoproteínas HDL, la dieta hipercalórica afecta sus niveles disminuyéndolos significativamente con respecto a las dietas no hipercalóricas (GC y GGIcN) $(p<0.028)$. La glucosamina contrarrestó este efecto para el grupo $(\mathrm{GH}+\mathrm{GlcN})$ aumentando sus niveles de HDL (Tabla 1).

El cálculo del índice LDL/HDL se mostró elevado para las dietas hipercalóricas (GH y GH (GICN), con diferencias significativas con respecto a las dietas no hipercalóricas (GC y GGlcN) $(p<0.004)$. Cabe destacar que este índice fue diferente entre los grupos con dietas hipercalóricas, disminuyéndose significativamente en el grupo con dieta hipercalórica más glucosamina $(\mathrm{GH}+\mathrm{Gl} \mathrm{cN})$ en relación al grupo hipercalórico $(p<0.02)$ (Tabla 1). Es observable que la glucosamina minimiza los efectos de las dislipidemias ocasionadas por una dieta hipercalórica sin mostrar diferencias significativas entre ellas ( $\mathrm{GH}$ versus $\mathrm{GH}+\mathrm{GlcN})(p>0.05)$. Sin embargo, es de destacar en este estudio que la administración de glucosamina aumenta los niveles de HDL, sin importar la dieta administrada, lo cual es importante en el control de dislipidemias ocasionadas por dietas hipercalóricas.

\section{Correlaciones grasa abdominal versus perfil lipídico y glucemia en suero}

Como se esperaba en relación a las correlaciones analizadas entre el contenido de grasa abdominal y las variables lipídicas estudiadas para los cuatro grupos de estudio, mientras mayor es el contenido de grasa abdominal corporal, mayor va a ser el contenido de Col T, AGL y TG así como el aumento de glucemia en sangre, disminuyéndose el HDL (Tabla 2) y siendo significativas todas las correlaciones $(p<0.01)$. Asimismo, al analizar las correlaciones de estas variables por grupos de estudio, tomando en cuenta los diferentes tratamientos administrados: dieta y glucosamina, y sumado al grupo control, se observó en la dieta hipercalórica que, al aumentar el contenido de grasa abdominal, aumenta a su vez el contenido de Col T, AGL, TG y glucemia en sangre, disminuyéndose el HDL $(p<0.001)$ y con aún mayores correlaciones encontradas entre los datos de estos dos grupos en estudio $(G C)+(G H)$ al total de los datos; sin

Tabla 1. Niveles del perfil lipídico: TG, Col T, HDL, LDL, VLDL (mg/dL) e indice LDL/HDL, de ratas machos sometidas a una dieta comercial (GC) e hipercalórica (GH); con administración de $500 \mathrm{mg} / \mathrm{kg}$ de glucosamina respectivamente (GGIcN y GH+GlcN) en el agua de beber, al final del tratamiento de 20 semanas.

\begin{tabular}{|c|c|c|c|c|}
\hline \multirow{2}{*}{ Perfil lipídico } & \multicolumn{2}{|c|}{ Dieta Comercial } & \multicolumn{2}{|c|}{ Dieta Hipercalórica } \\
\hline & Sin glucosamina (GC) & Con glucosamina (GGlcN) & Sin glucosamina $(\mathrm{GH})$ & Con glucosamina $(\mathrm{GH}+\mathrm{GlcN})$ \\
\hline $\mathrm{TG}(\mathrm{mg} / \mathrm{dL})$ & $75.28 \pm 6.21$ & $75.71 \pm 6.76$ & $165.71 \pm 11.43^{*} \gamma$ & $134.14 \pm 12.00^{\star} \gamma$ \\
\hline Col T (mg/dL) & $96.42 \pm 3.53$ & $107.85 \pm 5.14$ & $166.00 \pm 7.61^{\star} \gamma$ & $152.14 \pm 12.80^{*} \gamma$ \\
\hline $\mathrm{HDL}(\mathrm{mg} / \mathrm{dL})$ & $37.228 \pm 2.36$ & $38.42 \pm 1.88$ & $28.28 \pm 2.38^{*} \gamma$ & $33.57 \pm 1.68$ \\
\hline LDL (mg/dL) & $44.08 \pm 3.62$ & $54.28 \pm 5.82$ & $104.57 \pm 5.91^{*} \gamma$ & $91.74 \pm 4.30^{* \gamma}$ \\
\hline $\operatorname{VLDL}(\mathrm{mg} / \mathrm{dL})$ & $15.05 \pm 1.24$ & $15.14 \pm 1.35$ & $33.40 \pm 2.28^{*} \gamma$ & $26.82 \pm 2.40^{*} \gamma$ \\
\hline Indice LDL/HDL & $1.22 \pm 0.13$ & $1.44 \pm 0.18$ & $3.86 \pm 0.38^{* \gamma}$ & $2.79 \pm 0.23^{*} \gamma_{\phi}$ \\
\hline
\end{tabular}

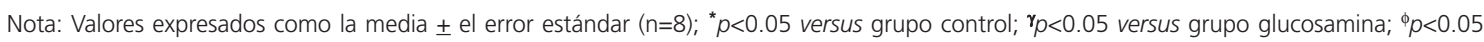
versus grupo hipercalórico. De acuerdo a la prueba analisis de varianza de dos vías.

GC: Grupo Controle; GH: Grupo Hipercalórico; GGIcN: Grupo Glucosamina; TG: Triglicerides; Col: Colesterol Total; HDL: High Density Lipoprotein; LDL: Low Density Lipoprotein; VDL: Very Low Density Lipoprotein. 
embargo, cuando se administra glucosamina junto a una dieta hipercalórica (GC) + ( GH+GGIcN), se minimizan los efectos de la dieta hipercalórica en los niveles HLD y glucemia en sangre contrarrestando su efecto; es decir, no hay una correlación entre el aumento de grasa abdominal y la disminución de HDL o aumento de glucemia en suero (Tabla 2). Cabe destacar que la glucosamina ayudó a aumentar el contenido de HLD, reguló la glucemia basal y ayudó a minimizar el contenido de grasa abdominal (Figura 2A).

La glucosamina administrada junto a una dieta normal mostró correlaciones mínimas, sin ser significativas entre el contenido de grasa abdominal y las variables lipídicas estudiadas y de glucemia en suero (Tabla 2) ( $p>0.05$ ).

En general se mostró en este estudio que la glucosamina no afectó ni provocó alteraciones en el perfil lipídico, aunque ayudó a minimizar el efecto de la dieta hipercalórica en las dislipidemias en ratas.

\section{DISCUSIÓN}

La investigación en modelos de roedores que imiten los cambios observados en los seres humanos es esencial ${ }^{11}$; uno de los principales criterios es que los modelos de roedores inicien todos los signos descritos en los seres humanos con síndrome metabólico, especialmente disglucemia y dislipemias, para la evaluación de la enfermedad y sus posibles tratamientos. Los efectos de las dietas hipercalóricas han sido ampliamente estudiados y relacionadas con la obesidad y otras patologías como las hiperlipidemias; en este estudio, los resultados de los grupos de ratas que recibieron una dieta hipercalórica no son la excepción: las ratas de los grupos GH y $\mathrm{GH}+\mathrm{Gl}$ cN tuvieron un aumento y ganancia de peso corporal (Figura 1A y B), aunque el consumo energético fue similar a los grupos con dieta normal GC y GGIcN; se ha reportado que la ingestión de grasas ha sido el eje del aumento de peso, por la interrelación compleja de factores como el apetito, la ingesta, la absorción de nutrimentos, reguladores neuroendocrinos, formas de almacenamiento de energía y gasto energético total, siendo la grasa un nutriente que se correlaciona positivamente con el aumento de peso ${ }^{26}$ y que se corresponde directamente con el aumento de la cantidad de grasa abdominal, efectos también observados para los grupos $\mathrm{GH}$ y GH+GlcN (Figura 2A). En este estudio, se ha descrito que, cuando el porcentaje de grasa en la dieta es elevado, se produce un desequilibrio entre la ingesta y la oxidación de la grasa que conduce a un aumento en el tamaño del tejido adiposo, hasta que los adipocitos se vuelven hipertróficos e hiperplásicos. Esto hace que la lipólisis en el tejido adiposo aumente y se liberen ácidos grasos libres ${ }^{27,28}$, con niveles de AGL también altos en este estudio (Figura 2B) y una correlación positiva de este aumento en los niveles de AGL y grasa

Tabla 2. Correlaciones entre el contenido de grasa abdominal y las variables de perfil lipídico: c-HDL, ColT, AGL y TG y glucemia de ratas machos sometidas a una dieta comercial (GC) e hipercalórica (GH); con administración de 500 mg/kg de glucosamina respectivamente ( $\mathrm{GGlcN}$ y GH+GlcN) en el agua de beber, al final del tratamiento de 20 semanas.

\begin{tabular}{|c|c|c|c|c|c|c|}
\hline \multirow{2}{*}{ Grupos de estudio relacionados } & & \multicolumn{5}{|c|}{ Variables evaluadas del perfil lípido en suero } \\
\hline & & $\mathrm{HDL}$ & $\mathrm{Col} \mathrm{T}$ & AGL & TG & Glucemia \\
\hline Todos los grupos de estudio $(n=28)$ & Grasa Abdominal & $-0.538^{* *}$ & $0.604^{* *}$ & $0.597^{* *}$ & $0.606^{* *}$ & $0.465^{*}$ \\
\hline Grupos GC y GH $(n=14)$ & Grasa Abdominal & $-0.686^{* *}$ & $0.793^{* *}$ & $0.819^{* *}$ & $0.837^{* *}$ & $0.793^{* *}$ \\
\hline Grupos GC y GGIcN $(n=14)$ & Grasa Abdominal & -0.205 & -0.142 & 0.068 & -0.257 & -0.236 \\
\hline Grupos GC y GH+GGIcN $(n=14)$ & Grasa Abdominal & 0.079 & $0.832^{* *}$ & $0.633^{*}$ & $0.623^{*}$ & 0.331 \\
\hline
\end{tabular}

Nota: ${ }^{*} p<0.05$ versus grupo control; $p<0.05$ versus grupo glucosamina. De acuerdo a la prueba Pearson.

GC: Grupo Controle; GH: Grupo Hipercalórico; GGlcN: Grupo Glucosamina; HDL-c: High Density Lipoprotein-cholesterol; Col: Colesterol Total; AGL: Acidos Graxos Libres; TG: Triglicerides. 
abdominal $(R=0.597, p<0.01)$ y TG $(R=0.606$, $p<0.01$ ) (Tabla 2) circulantes, dada la dieta hipercalórica, siendo el hipotálamo el que censa las concentraciones de nutrientes en suero y la cantidad de grasa corporal. Este sistema está considerando como un sensor celular de disponibilidad de energía ${ }^{29}$; es decir, se suma a su capacidad de respuesta frente a los niveles de hormonas circulantes la respuesta del cerebro directamente ante la presencia de nutrientes tales como la glucosa, los ácidos grasos y los aminoácidos, siendo aparentemente una evidencia de que informan al sistema nervioso central acerca del estado energético con el propósito de inducir cambios en el comportamiento apetitivo y el balance energético ${ }^{30}$. Las alteraciones de aumento en el consumo energético, junto con la disminución en el gasto energético, sugieren una alteración en los mecanismos efectores que regulan tanto el consumo y el control en el aumento de peso, en el cual diversos factores están vinculados a su desarrollo, como la ingestión hipercalórica ${ }^{31,32}$.

Dentro de las alteraciones del aumento de peso por una dieta hipercalórica se reporta la intolerancia a la glucosa, lo que a su vez explicaría el aumento progresivo de glucemia basal a lo largo del tiempo, alteración observada para el grupo $\mathrm{GH}$, que incluso llega a valores limítrofes: $119 \mathrm{mg} /$ dL (Figura 2C). Se observa también que el aumento de AGL puede influir en la captación de glucosa estimulada por insulina ${ }^{33,34}$, el cual provoca una reducción del metabolismo de glucosa ${ }^{35}$ producida por mecanismos como disminución de la captación y utilización de la glucosa, inhibición del ciclo de Krebs, de secreción y receptores de insulina, disminución de transportadores GLUT4 ${ }^{36}$ y estimulación de la lipasa sensible a hormonas que favorece la lipólisis de los TG en tejido adiposo, aumentando los AGL y la glucemia basal ${ }^{37,38}$.

El aumento de TG (hipertrigiceridemia) circulantes adquiridos por las dietas, fuertemente relacionado con la obesidad visceral ${ }^{2}$, puede relacionarse también con bajos niveles de HDL; esto recibe el nombre de dislipidemia aterogénica, siendo característica del síndrome metabólico; a su asociación subsecuente con la hipercolesterolemia se la denomina dislipidemia mixta. En este estudio se observó un aumento del colesterol Col T en los grupos con dieta hipercalóricas $\mathrm{GH}$ y GH+GlcN, con disminución del colesterol de alta densidad HDL (Tabla 1), inferido por la misma dieta hipercalórica administrada durante el tratamiento, correlacionados con un aumento de la grasa abdominal positivamente para $\mathrm{Col} T(\mathrm{R}=0.604$, $p<0.001)$ y negativamente para $\mathrm{HDL}(R=-0.538$, $p<0.001)$. Este fenómeno se establece principalmente por un desequilibrio entre el ingreso y el gasto energético que resulta en una acumulación excesiva de grasa, particularmente en el tejido adiposo visceral, que muchas veces se asocia con un síndrome metabólico ${ }^{4,39}$; esta aterogenicidad por la hipertrigliceridemia fue observada principalmente gracias a que los niveles de HDL también pierden colesterol y adquieren TG, los cuales son hidrolizados por la lipasa hepática, con reducción de $\mathrm{HDL}$, que son las partículas eficientes en el transporte reverso del colesterol y el aumento de $H D L$, partículas pequeñas y pobres en colesterol con escasa capacidad antiaterogénica; las lipoproteínas ricas en TG y enriquecidas en colesterol, resultantes de este aumento del intercambio lipídico, también son aterogénicas, ya que no se captan bien por los receptores hepáticos; por su parte, los niveles de colesterol de baja densidad se enriquecen en TG, lo que las convierte en un buen sustrato para la lipasa hepática, que hidroliza los TG, formando moléculas de LDL más densas y pequeñas, con un aumento relativo del contenido de apo $B$ respecto al de colesterol. Estas $L D L$ anómalas son, en definitiva, más aterogénicas que las LDL normales ${ }^{16,40}$. Al calcular los niveles de LDL, VLDL y el índice LDL/HDL, con los datos obtenidos sobre los análisis serológicos de TG, HDL y Col T, estos aumentaron para los grupos con dieta hipercalórica $\mathrm{GH}$ y $\mathrm{GH}+\mathrm{GlcN}$, alcanzando niveles de riesgo aterogénico como parte de las dislipidemias (Tabla 1), siendo diferentes estadísticamente con 
respecto al grupo GC y el grupo con glucosamina GGlcN ( $p<0.001)$.

Cuando se detectan las alteraciones provocadas principalmente por las dietas hipercalóricas, pueden usarse diferentes sustancias para controlar la dislipidemia aterogenica y reducir los riesgos metabólicos y cardiovasculares como fibratos, estatinas, niacina y tiazolidinadionas ${ }^{41}$; sin embargo, en este estudio se probó el uso de la glucosamina a través de los reportes en los que se menciona que esta sustancia presenta efectos anti-obesidad, así como posibles efectos para disminuir los niveles de TG ${ }^{19,42}$. Se observa por otro lado que la glucosamina ayuda a disminuir la ganancia de peso corporal en el grupo de ratas con dieta hipercalórica ( $\mathrm{GH}+\mathrm{GlcN}$ ) cuando se administra por 15 semanas a una dosis de 500 $\mathrm{mg} / \mathrm{kg} / \mathrm{día}$, siendo estadísticamente diferente el peso del grupo de ratas que solo recibió una (GH) $(p<0.05)$ (Figura 1A y B), minimizando el contenido de grasa visceral y $A G L$ sin presentar diferencia significativa (Figura 2A y B), regulando los valores de glucemia para este grupo de ratas, llevándolos a valores normales (Figura 2C).

Con respecto al perfil lipídico, los niveles de TG, LDL, VLDL e índice LDL/HDL fueron minimizados en ratas con dieta hipercalórica cuando se administró glucosamina ( $\mathrm{GH}+\mathrm{GlcN})$, aunque la alteración y el aumento de dislipidemias presentados fue estadísticamente diferente con respecto al GC, lo cual mostró que la glucosamina minimiza los efectos de la dieta hipercalórica; a pesar de ello hacen falta más estudios para que pueda ser considerado como un tratamiento alternativo en contra de las dislipidemias provocadas por dieta hipercalórica. Así y todo, es importante mencionar que el uso de derivados de glucosamina en los estudios en modelos animales en el metabolismo de lípidos es reciente y propone la hipótesis que esta sustancia favorece el metabolismo celular de los lípidos, principalmente AGL y la lipotoxicidad que éstos producen ${ }^{43}$, posiblemente por efecto a través de la regulación y activación de la proteincinasa estimulada por el
Adenosina Monofosfato (AMPK) ${ }^{42}$ y de la sobreexpresión de la vía de las hexosaminas estimulada por glucosamina, en la oxidación de ácidos grasos libres ${ }^{44}$, ya que la AMPK contribuye a la disminución o aumento de los niveles de Malonil CoA, inhibidor directo inhibidor de carnitina palmitoil transferasa tipo 1 (CPT1) que regula el transporte de ácidos grasos al interior de la mitocondria; es decir, se induce un aumento de la oxidación de $A G L$ ante una oferta de lípidos (dieta hipercalórica), sugiriendo que la actividad del AMPK es bloquear la formación de acetil-CoA carboxilasa e inducir un efecto lipooxidativo y estimular la mayor utilización de la glucosa ${ }^{45}$. Así, la activación de AMPK, podría ser la clave por la cual la glucosamina minimiza los efectos de la dieta hipercalórica dentro el metabolismo de carbohidratos y lípidos, retrasando los efectos de dislipidemias e incluso regulando la glucemia, como se observó en este estudio para el grupo que se le administró glucosamina junto a una dieta hipercalórica.

Cabe también mencionar que en este estudio la glucosamina reguló los niveles de HDL para la dieta hipercalórica ( $\mathrm{GH}+\mathrm{GlcN})$, lo que remarca una importancia, ya que la regulación de HDL es el nuevo blanco terapéutico en los niveles plasmáticos del colesterol transportado en este tipo de lipoproteínas. Tanto estudios experimentales, epidemiológicos, genéticos y clínicos indican que los niveles de HDL se correlacionan en forma independiente e inversa con la presencia de dislipidemias y la enfermedad cardiovascular, indicando que las HDL constituyen un factor protector antiaterogénico ${ }^{46}$.

Los grupos que fueron alimentados con una dieta no hipercalórica no presentaron aumento en los niveles de perfil lipídico, manteniéndose en valores normales. Y al administrarse glucosamina junto a esta dieta no se alteraron las mediciones realizadas.

\section{CONCLUSIÓN}

En conclusión, este estudio mostró que la glucosamina ayuda a controlar el aumento de 
peso y la glucemia en sangre así como minimiza las dislipidemias provocadas por la dieta hipercalórica en ratas, favoreciendo el aumento de colesterol de alta densidad, y sugiriendo que deben realizarse estudios comparativos y complementarios con algún tipo de fármaco convencional para el tratamiento de dislipidemias ocasionado por dietas hipercalóricas. La glucosamina administrada de forma exclusiva a una dieta comercial, no presenta ningún efecto adverso o eficaz en el peso corporal y perfil lipídico de la rata.

\section{A GRADECIMIENTOS}

Se agradece a la Secretaria de Investigación y Posgrado del Instituto Politécnico Nacional por el financiamiento y apoyo brindado para la realización de este estudio (SIP-20131233).

\section{COLABORACIONES}

C BARRIENTOS ALVARADO Búsqueda de información, elaboración del protocolo y formación de los grupos de investigación, experimentación, experimento, base de datos, análisis estadístico y realización de manuscrito y reporte final para informe de investigación. Determinación del posible efecto protector de la glucosamina sobre el perfil lipídico. MAS CÁRDENAS OSCOY Búsqueda de información, elaboración del protocolo y formación de grupos de investigación, realización del manuscrito e informe final. J VÁZQUEZ SÁNCHEZ Apoyo experimental, base de datos y elaboración del manuscrito e informe final. 0 GARRIDO ACOSTA Apoyo experimental, base de datos, análisis estadístico y realización de manuscrito. L ANGUIANO ROBLEDO Apoyo experimental, base de datos, análisis estadístico y realización del manuscrito.

\section{REFERENCIAS}

1. Siriwardhana N, Kalupahana NS, Cekanova M, Lemieux M, Greer B, et al. Modulation of adipose tissue inflammation by bioactive food compounds. J Nutr Biochem. 2013; 24(4):613-23. doi: 10.1016/ j.jnutbio.2012.12.013

2. Carr MC, Brunzell JD. Abdominal obesity and dyslipidemia in the metabolic syndrome:
Importance of type 2 diabetes and familialcombined hyperlipidemia in coronary artery disease risk. J Clin Endocrinol Metab. 2004; 89(6):2601-7. doi: 10.1210/jc.2004-0432

3. Brunzell JD, Ayyobi AF. Dyslipidemia in the metabolic syndrome and type 2 diabetes. Am J Med. 2003; 115(S8):S24-8. doi: 10.1016/j.amjmed.2003.0 8.011

4. Lemieux I, Pascot A, Couillard C, Lamarche B, Tchernof $A$, Almeras $N$, et al. Hypertriglyceridemic waist: A marker of the atherogenic metabolic triad (hyperinsulinemia; hyperapolipoprotein B; small, dense LDL) in men? Circulation. 2000; 102(2):179-84. doi: 10.1161/01.CIR.102.2.179

5. Aguilar SCA, Rojas R, Gómez PFJ, Valles V, Rios JM, Franco $A$, et al. High prevalence of metabolic syndrome in México. Arch Med Res. 2004; 35(1):76-81. doi: 10.1016/j.arcmed.2003.06.006

6. Kalupahana NS, Moustaid-Moussa N, Claycombe KJ. Immunity as a link between obesity and insulin resistance. Mol Aspects Med. 2012; 33(1):26-34. doi: 10.1016/j.mam.2011.10.011

7. Durrington P. Dyslipidaemia. Lancet. 2003; 362(9385):717-31. doi: 10.1016/S0140-6736(03) 14234-1

8. Wang S, Moustaid-Moussa N, Chen L, Mo H, Shastri A, Su R, et al. Novel insights of dietary polyphenols and obesity. J Nutr Biochem. 2014; 25(1):1-18. doi: 10.1016/j.jnutbio.2013.09.001

9. Velázquez MO, Rosas PM, Lara EA, Pastelín HG, Hernández C, Sánchez A, et al. Prevalencia e interrelación de enfermedades crónicas no transmisibles y factores de riesgo cardiovascular en México. Arch Cardiol Mex. 2003; 73(1):62-77.

10. Massiera F, Barbry P, Guesnet P, Joly A, Luquet S, Moreilhon-Brest $C$, et al. A Western-like fat diet is sufficient to induce a gradual enhancement in fat mass over generations. J Lipid Res. 2010; 51(8):2352-236. doi: 10.1194/Jlr.M006866

11. Panchal SK, Brown L. Rodent models for metabolic syndrome research. J Biomed Biotechnol. 2011; 20011:351982. doi: 10.1155/2011/351982

12. Rolland V, Roseau S, Fromentin G, Nicolaidis S, Tomé $D$, Even PC. Body weight, body composition, and energy metabolism in lean and obese zucker rats fed soybean oil or butter. Am J Clin Nutr. 2002; 75(1):21-30.

13. Panchal SK, Poudyal $H$, lyer A, Nazer $R$, Alam A, Diwan $V$, et al. High-carbohydrate, high-fat dietinduced metabolic syndrome and cardiovascular remodeling in rats. J Cardiovasc Pharmacol. 2011; 57(5):611-24. doi: 10.1097/FJC.0b013e3181f eb90a 
14. Lomba A, Milagro Fl, Garcia-Diaz DF, Marti A, Campion J, Martinez JA. Obesity induced by a pairfed high fat sucrose diet: Methylation and expression pattern of genes related to energy homeostasis. Lipids Health Dis. 2010; 9:60. doi: 10.1186/1476-511X-9-60

15. Sever P, Dahlof B, Poulter N, Wedel H, Beevers G, Caulfield $\mathrm{M}$, et al. Prevention of coronary and stroke events with atorvastatin in hypertensive patients who have average or lower-than-average cholesterol concentrations, in the Anglo-Scandinavian Cardiac Outcomes Trial-Lipid Lowering Arm (ASCOT-LLA): A multicenter randomized trial. Lancet. 2003; 361(9364):1149-58. doi: 10.1016/S0140-673 6(03)12948-0

16. Kwiterovich PO. The metabolic pathways of highdensity lipoprotein, low-density lipoprotein, and triglycerides: A current review. Am J Cardiol. 2000; 86(12A):L5-10. doi: 10.1016/S0002-9149(00)0 1461-2

17. McAlindon TE, LaValley MP, Gulin JP, Felson DT. Glucosamine and chondroitin for treatment of osteoarthritis: A systematic quality assessment and meta-analysis. Jama. 2000; 283(11):1469-75. doi: 10.1001/jama.283.11.1469

18. Bondiolotti G, Bareggi SR, Frega NG, Strabioli S, Cornelli U. Activity of two different polyglucosamines, L112 and FF45, on body weight in male rats. Eur J Pharmacol. 2007; 567(1-2):155-8.

19. Bondiolotti G, Cornelli U, Strabbioli RS, Frega NG, Cornelli M, Bareggi S. Effect of a polyglucosamine on the body weight of male rats: Mechanisms of action. Food Chem. 2011; 124(3):978-82. doi: 10.1016/j.foodchem.2010.07.039

20. Magrans CT, Wilborn C, Rasmussen C, Ferreira M, Greenwood L, Campbell B, et al. Effects of diet type and supplementation of glucosamine, chondroitin, and MSM on body composition, functional status, and markers of health in women with knee osteoarthritis initiating a resistance-based exercise and weight loss program. J Int Soc Sports Nutr. 2011; 8(1):8. doi: 10.1186/1550-2783-8-8

21. Lamiaa A, Barakat A. Hypolipidemic and antiatherogenic effects of dietary chitosan and wheat bran in high fat-high cholesterol fed rats. Aust J Basic Appl Sci. 2011; 5(10):30-7.

22. Muurling M, Mensink RP, Pijl H, Romijn JA, Havekes LM, Voshol PJ. A fish oil diet does not reverse insulin resistance despite decreased adipose tissue TNFalpha protein concentration in ApoE-3* Leiden mice. J Nutr. 2003; 133(11):3350-5.

23. Anderson JW, Nicolosi RJ, Borzelleca JF. Glucosamine effects in humans: A review of effects on glucose metabolism, side effects, safety considerations and efficacy. Food Chem Toxicol.
2005; 43(2):187-201. doi: 10.1016/j.fct. 2004.1 1.006

24. Keita H, Ramírez-San Juan E, Paniagua-Castro N, Garduño-Siciliano L, Quevedo L. The long-term ingestion of a diet high in extra virgin olive oil produces obesity and insulin resistance but protects endothelial function in rats: A preliminary study. Hady Diabetol Metab Synd. 2013; 5:53 doi: 10.1 186/1758-5996-5-53

25. Friedewald WT, Levy RI, Fredrickson DS. Estimation of the concentration of low-density lipoprotein cholesterol in plasma, without use of the preparative ultracentrifuge. Clinl Chem. 1972; 18(6):499-502.

26. Sánchez-Lasheras C, Könner AC, Brüning JC. Integrative neurobiology of energy homeostasisneurocircuits, signals and mediators. Front Neuroendocrinol. 2010; 31(1):4-15. doi: 10.1016/j. yfrne.2009.08.002

27. Laye MJ, Thyfault JP, Stump CS, Booth FW. Inactivity induces increases in abdominal fat. J Appl Physiol. 2007; 102(4):1341-7.

28. Fernández-Quintela A, Churruca I, Portillo MP. The role of dietary fat in adipose tissue metabolism. Public Health Nutr. 2007; 10(10A):1126-31.

29. Könner AC, Klöckener T, Brüning JC. Control of energy homeostasis by insulin and leptin: Targeting the arcuate nucleus and beyond. Physiol Behav. 2009; 97(5):632-8. doi: 10.1016/j.physbeh.200 9.03 .027

30. Marty N, Dallaporta M, Thorens B. Brain glucose sensing, counterregulation, and energy homeostasis. Physiology. 2007; 22:241-51.

31. Stein CJ, Colditz GA. The epidemic of obesity. J Clin Endocrinol Metab. 2004; 89(6):2522-5.

32. Hensrud DD. Diet and obesity. Curr Opin Gastroenterol. 2004; 20(2):119-24.

33. Stannard SR, Johnson NA. Insulin resistance and elevated triglyceride in muscle: More important for survival than thrifty genes? J Physiol. 2004. 554(Pt3):595-607. doi: 10.1113/jphysiol.2003.0 53926

34. Yu YH, Ginsberg HN. Adipocyte signaling and lipid homeostasis: Sequelae of insulin-resistant adipose tissue. Circ Res. 2005; 96(10):1042-52. doi: 10.1 161/01.RES.0000165803.47776.38

35. Boden G. Obesity, insulin resistance and free fatty acids. Curr Opin Endocrinol Diabetes Obes. 2011; 18(2):139-43. doi: 10.1097/MED.0b013e32834 44b09

36. Kahn SE, Hull RL, Utzschneider KM. Mechanisms linking obesity to insulin resistance and type 2 diabetes. Nature. 2006; 444(7121):840-6. 
37. Arner P. Human fat cell lipolysis: Biochemistry, regulation and clinical role. Best Pract Res Clin Endocrinol Metab. 2005; 19(4):471-82. doi: 10.1016/j.beem.2005.07.004

38. Frayn KN. Obesity and metabolic disease: Is adipose tissue the culprit? Proc Nutr Soc. 2005; 64(1):7-13. doi: org/10.1079/PNS2004403

39. Lemieux I, Poirier $P$, Bergeron J, Alméras N, Lamarche B, Cantin B, et al. Hypertriglyceridemic waist: A useful screening phenotype in preventive cardiology? Can J Cardiol. 2007; 23(Suppl. B):23B-31B.

40. Fujimoto VY, Kane JP, Ishida BY, Bloom MS, Browne RW. High-density lipoprotein metabolism and the human embryo. Hum Reprod. 2010; 16(1):20-38. doi: 10.1093/humupd/dmp029

41. Expert Panel on Detection, Evaluation, and Treatment of High Blood Cholesterol in Adults. Executive Summary of the Third Report of the National Cholesterol Education Program (NCEP) Expert Panel on Detection, Evaluation, and Treatment of High Blood Cholesterol in Adults (Adult Treatment Panel III). Jama. 2002; 285(2):2486-97. doi: 10.1001/jama.285.19.2486
42. Kong CS, Kim JA, Kim SK. Anti-obesity effect of sulfated glucosamine by AMPK signal pathway in 3T3-L1 adipocytes. Food Chem Toxicol. 2009; 47(10):2401-06. doi: 10.1016/j.fct.2009.06.010

43. Lechleitner M. Mitochondrial function role in insulin resistance and lipid metabolism. Acta Med Austriaca. 2004; 31(4):115-9.

44. Luo B, Parker GJ, Cooksey RC, Soesanto Y, Evans $\mathrm{M}$, Jones $\mathrm{D}$, et al. Chronic hexosamine flux stimulates fatty acid oxidation by activating AMP-activated protein kinase in adipocytes. J Biol Chem. 2007; 282(10):7172-80. doi: 10.1074/jbc.M607362200

45. Unger RH. Minireview: Weapons of lean body mass destruction: The role of ectopic lipids in the metabolic syndrome. Endocrinology. 2003; 144(12):5159-65. doi: 10.1210/en.2003-0870.51 59

46. Lewis GF, Rader DJ. New insights into the regulation of HDL metabolism and reverse cholesterol transport. Circ Res. 2005; 96(12):1221-32.

Recibido: 27/2/2014

Versión final: 15/7/2014

Aprobado: 9/9/2014 
